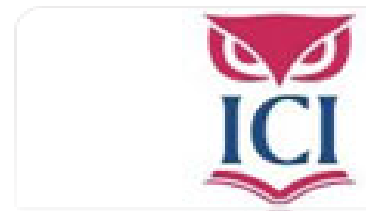

IUS. Revista del Instituto de Ciencias Jurídicas de Puebla A.C.

ISSN: 1870-2147

revista.ius@hotmail.com

Instituto de Ciencias Jurídicas de Puebla A. C.

México

Martínez Sierra, José Manuel

LOS SISTEMAS ELECTORALES DESDE UNA PERSPECTIVA COMPARADA

IUS. Revista del Instituto de Ciencias Jurídicas de Puebla A.C., núm. 18, 2006, pp. 20-38 Instituto de Ciencias Jurídicas de Puebla A. C.

Puebla, México 


\section{LOS SISTEMAS ELECTORALES DESDE UNA PERSPECTIVA COMPARADA}

Dr. José Manuel Martínez Sierra*

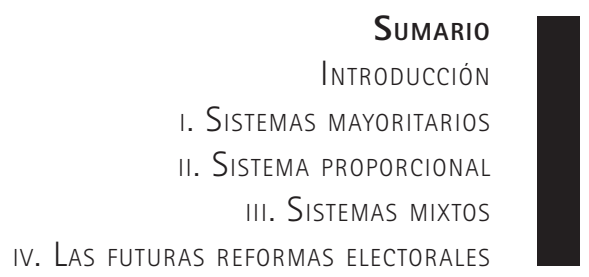

\section{INTRODUCCIÓN}

Los sistemas electorales son concebidos en la teoría democrática como el único instrumento posible para determinar quiénes han de ser los representantes del pueblo y en qué número han de representarlo. Esta idea resume el sentido de los sistemas electorales en las democracias representativas. Dada la imposibilidad de realizar una democracia directa, por los motivos materiales de eficacia y eficiencia sentados por la teoría clásica de la representación, la democracia constitucional hegemónica es la representativa y ésta se basa en:

Un órgano que sirva como punto de encuentro entre los representantes y la soberanía popular, entre los diputados y la población: el Parlamento.

Un sistema electoral que articule la representación; idealmente a través de la consecución de la igualdad perfecta, es decir, que el voto de cada elector tenga un mismo peso o traducción en peso parlamentario.

De la relación de estas dos premisas devendría, para el sistema electoral, la carga de asegurar que los representantes que se dan cita en el Parlamento guarden una relación cuantitativa directamente proporcional con lo dictado por los electores a través del sufragio. En definitiva, que

* El autor es profesor titular de derecho constitucional en la Facultad de Derecho de la Universidad Complutense de Madrid. 
el Parlamento sea una réplica a tamaño reducido de la soberanía popular movilizada electoralmente y a la que representa.

El sistema electoral que consigue esto se denomina "sistema proporcional integral”. Pero como bien ha demostrado la politología, este sistema no se da en ningún país. Por nuestra parte, trataremos los motivos fundamentales por los que los sistemas electorales se separan de este óptimo democrático.

\section{Sistemas mayoritarios}

Dentro de estos sistemas podemos distinguir los que son a una vuelta o a dos. La característica común a ambos es que eliminan literalmente a las minorías locales e infrarrepresentan a las minorías nacionales. La distribución real de votos y el reparto posterior de escaños entre los partidos no guarda una relación ni siquiera aproximada.

\section{I.1. Sistema mayoritario a una vuelta}

Es el sistema utilizado en el Reino Unido, bajo la lógica del the first past the post. Es decir, en cada circunscripción se presentará un número indeterminado e ilimitado de candidatos, pero cada circunscripción dará únicamente un asiento o escaño; dicho escaño será para el candidato que obtenga mayor número de votos. El resto de votos se descarta, no sirviendo para ulteriores recuentos; son votos perdidos.

Si en una circunscripción el candidato A obtiene 70,000 votos; el B, 65,000 y el C, 40,000 votos, el candidato A ganará el escaño de la circunscripción con 70,000 votos, quedando desechados los 105,000 restantes. Este ejemplo corresponde al escrutinio uninominal existente en Reino Unido. Una variante de este sistema es el escrutinio de lista, como el denominado "turco", donde, siguiendo el ejemplo anterior, la lista A se llevaría todos los escaños de la circunscripción, en tanto lo que se vota no es a una persona, sino a una lista de partido y otorgándose en consecuencia varios escaños por circunscripción. Siguiendo este procedimiento, en 1934, el Partido Demócrata de Turquía obtuvo el 93\% de los escaños y solo el 58\% de los votos.

Volviendo al sistema uninominal británico hay que destacar que ha producido desequilibrios muy notables en diversas elecciones (vid. gráfico 1), aunque no son los más descaradamente desproporcionados de su 
historia. En 1983, el Partido Liberal, con el 25.4 de los votos obtuvo 23 escaños en el Parlamento, mientras que el Partido Laborista, con el 27.6 de los votos, obtuvo 209 escaños. En 31 años, de 1974 a 2005, se han dado gobiernos monocolores, que gobiernan con mayorías absolutas en el Parlamento sin sobrepasar en ningún momento el 42.4\% de los votos, y llegando a gobernar con mayoría los laboristas, en el periodo 1974 a 1979, con sólo el 39.2\% de los votos. Si contabilizamos el número de británicos que apoyan al partido en el gobierno con sus votos y los que apoyan a los restantes, tendremos un sistema "minoritario" (valga esta expresión, que se asemeja más a la realidad que la de "mayoritario"). Así, en 1983 y en el 1987 los conservadores obtuvieron 13,012,612 y 13,763,134 de votos, de un total de 30,683,484 y 32,534,657 de votos emitidos, respectivamente. Si el total de la población británica oscilaba entre 56 y 57 millones de personas, se puede decir que los gobiernos tienen un apoyo poblacional muy minoritario. Incluso en 1951, cuando el Partido Conservador obtuvo 321 escaños gracias al 48\% de los votos, el Partido Laborista sacó 295 escaños con el 48.8\% de los votos, lo cual nos demuestra hasta dónde puede llegar el sistema mayoritario a una vuelta.

La tendencia general de este sistema es que el partido ganador obtenga normalmente un porcentaje de escaños muy superior al porcentaje de sufragios. El segundo partido estaría representado en proporciones inferiores al primero. Y el tercer partido de dimensión nacional, que desde el primer cuarto del siglo es el Liberal, estaría infrarrepresentado.

Pero el sistema mayoritario no sólo tergiversa los sufragios emitidos, sino que también tergiversa la intención de voto, rompiendo con la aproximación individualista y liberal del voto. Esto se produce porque muchos electores se ven obligados a realizar lo que los ingleses llaman un "voto táctico". Dicho voto consiste en que el elector no vota al partido que considera adecuado porque no tiene posibilidades de ganar en su circunscripción, sino a otro partido, ya porque sea su segunda preferencia o ya simplemente, para perjudicar al partido que el elector no desea que gane. Así, las encuestas que se publicaron en abril de 1992, antes de las elecciones inglesas, daban al Partido Liberal una intención de voto del $40 \%$ que luego descendió a otra del 20\%, para finalmente obtener un 18\% (vid. gráfico 1). Esto confirma la primera ley de Duverger: el sistema mayoritario tiende al bipartidismo. ${ }^{1}$

${ }^{1}$ M. Duverger, Los partidos politicos, Fondo de Cultura Económica, México, 1981. 
También se puede observar que es muy difícil que surjan partidos temporales o de moda, o que un nuevo partido suplante a otro mayoritario, ya que el sistema electoral ha polarizado a la sociedad en dos tendencias que refuerzan el papel de los partidos mayoritarios, que tienen una tradición histórica vinculada y desarrollada en los territorios de muchas circunscripciones.

Gráfico 1: Sistema electoral mayoritario. Gran Bretaña: elecciones generales del 9 de abril de 1992

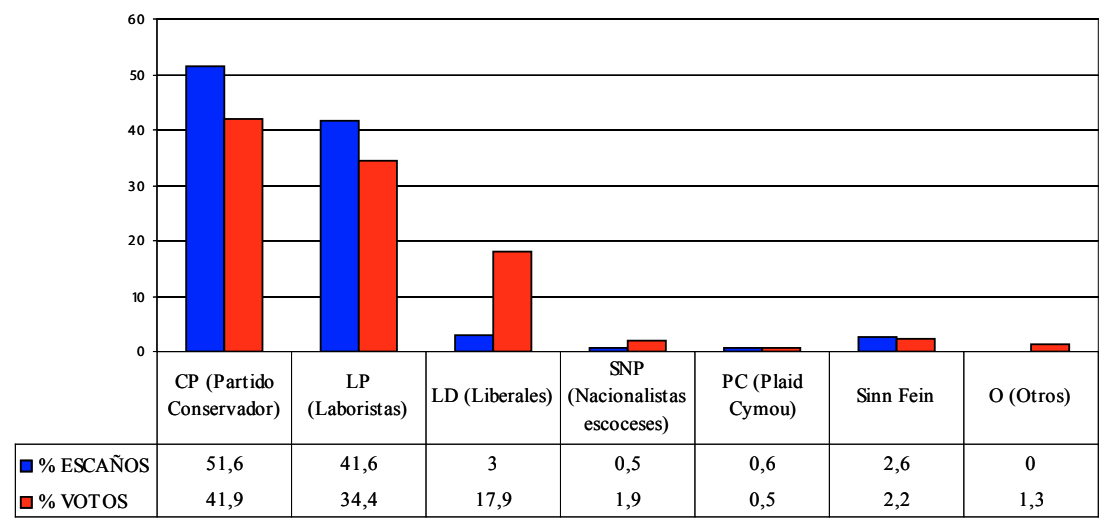

Fuente: Elaboración propia.

Gráfico 1-bis: Sistema electoral mayoritario. Gran Bretaña: elecciones generales del 5 de mayo de 2005

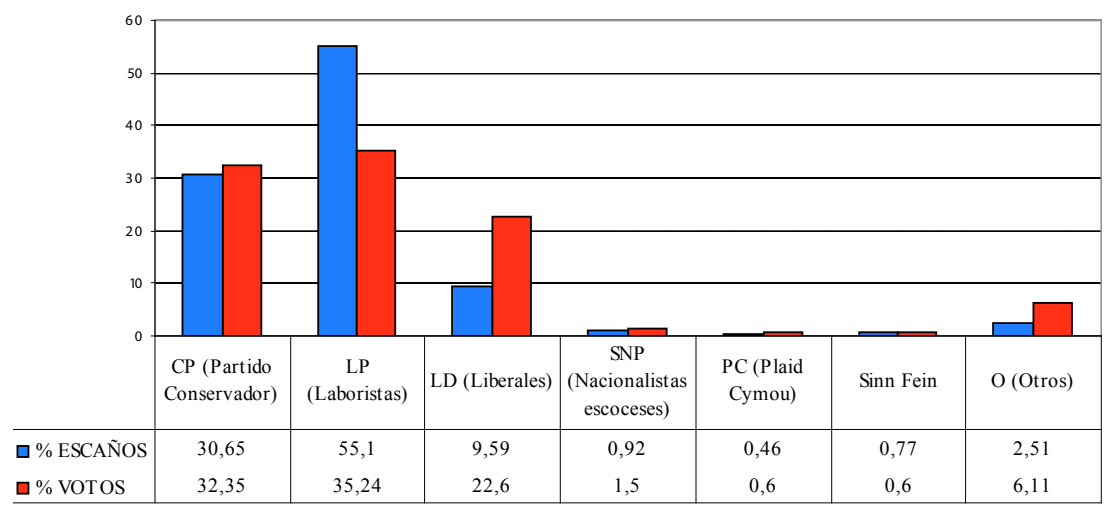

Fuente: Elaboración propia. 


\section{I.2. Sistema mayoritario a dos vueltas}

Es el que actualmente se da en Francia y el predominante durante la V República. A diferencia del sistema mayoritario a una vuelta, en el sistema mayoritario a dos vueltas, para obtener un escaño en el escrutinio es necesario tener la mitad de los votos más uno en la circunscripción (en la primera vuelta), es decir, la mayoría absoluta; en caso contrario, se procede a una segunda vuelta en la cual basta con alcanzar la mayoría simple. En esta segunda vuelta el sistema funciona exactamente como en el sistema mayoritario a una vuelta.

En líneas generales, se puede decir que los fenómenos descritos en el sistema a una vuelta se atenúan en el escrutinio a dos vueltas, pero no por ello se anulan las injusticias de este sistema, mayoritario al fin y al cabo. Las desproporciones son mayores cuanto más débil es el número de escaños previsto a la salida de la primera vuelta, es decir, a la salida de la mayoría absoluta, ya que en este caso sólo se echan a perder el 49\% o menos de los votos. En la segunda vuelta, tras las renuncias y retiradas de candidatos mal situados, quedan enfrentadas dos opciones, la primera y la segunda. Ciertas fuerzas políticas se encuentran entonces mal representadas. Por ejemplo, en 1968 el Partido Comunista, con el 20\% de los sufragios, sólo obtuvo el 7\% de los escaños, mientras la coalición de la UDR con los republicanos, con el 44\% de los sufragios, ganó el 70\% de los escaños. Como apunta Cotteret, una de los mayores defectos del sistema, desde el punto de vista cualitativo, radica en la naturaleza de los acuerdos que se producen entre las dos vueltas, para planificar la renuncia de un partido en beneficio de otro, con lo que perjudican gravemente a terceros. Ejemplo de esto es el perjuicio que se causa a las segundas o terceras opciones nacionales, en algunas circunscripciones donde incluso pueden ser mayoritarias en la primera vuelta. Así mientras que en la primera vuelta tiene la mayoría, en la segunda la pierde por la renuncia de unos partidos en favor de otros. Esto se produce principalmente entre la UDF y RPR. Esta relación se ha dado también entre el Partido Socialista y el Partido Comunista, aunque hoy el pacto se ve debilitado por el declive progresivo del PCF.

Duverger, llevado por estos hechos, afirma que este sistema tiende a un multipartidismo atemperado por alianzas. El sistema da oportunidad a muchos partidos a probar suerte en la primera vuelta, pero, sin embargo, en la segunda han de reagruparse por afınidad ideológica o de interés. Así, siguiendo el ejemplo anterior, si en la primera vuelta el RPR se hace con 
50,000 votos en una circunscripción, la UDF 40,000 y el FN 70,000 votos; en la segunda vuelta si el RPR y la UDF no se ponen de acuerdo, el representante será del FN. Ello obliga a aliarse a dos partidos para derrotar a un tercero. El proceso se produce normalmente por imperativo legal, que obliga a que solamente los dos partidos más votados en la primera vuelta concurran a la segunda; pero una vez en la segunda vuelta las posibilidades son varias: puede haber retirada o desistimiento de uno de los dos partidos en la segunda vuelta; o puede haber el apoyo a uno de los dos candidatos en liza por parte de los partidos que quedaron descartados para la segunda vuelta.

Quienes dirigen la operación por supuesto, son las élites partitocráticas, que no tienen en cuenta a los electores que en la primera vuelta indicaron claramente su preferencia de voto por un partido. Si en la segunda vuelta se produce la marginación directa, por el sistema electoral, o el desistimiento (marginación indirecta) del partido, el votante tendrá que realizar el mecanismo del voto útil o táctico, o abstenerse.

Los efectos del escrutinio a dos vueltas favorecen algo menos a los grandes partidos que el sistema mayoritario a una vuelta. También polariza algo menos a los grandes partidos, pero fomenta coaliciones contra la sociología electoral francesa, al menos tal y como se refleja en una primera vuelta que plasma más claramente las intenciones de voto de los electores. Así, el multipartidismo real se ve atemperado por alianzas de fuerzas mayoritarias, alianzas que dificultan gravísimamente la obtención de escaños por fuerzas minoritarias.

En 1986, con un sistema proporcional, el FN afloró en la Asamblea Nacional francesa con 35 escaños. En 1988 se volvió al sistema mayoritario a doble vuelta y el FN, con el mismo porcentaje de votos (el 9.65), obtuvo un escaño. Observamos pues que, si bien el sistema proporcional del 86 perjudica a los partidos minoritarios, ya que el 9.65 de los votos sólo otorgó al FN el 6.04\% de los escaños, el sistema mayoritario a doble vuelta tiende a hacerle extraparlamentario, como ocurrió en las últimas elecciones de los noventa, donde, pese a aumentar en $2.7 \%$ su porcentaje de votos, perdió el escaño del 87 (vid. gráfico 2). Con ello, los partidos mayoritarios franceses consiguen marginar a las fuerzas políticas minoritarias, frenando su crecimiento al apartarlas de las ventajas de estar representadas en el Parlamento (financiación, publicidad, información, etc.). 
Gráfico 2: Sistema mayoritario a dos vueltas. Francia: elecciones generales del 28 de marzo de 1993

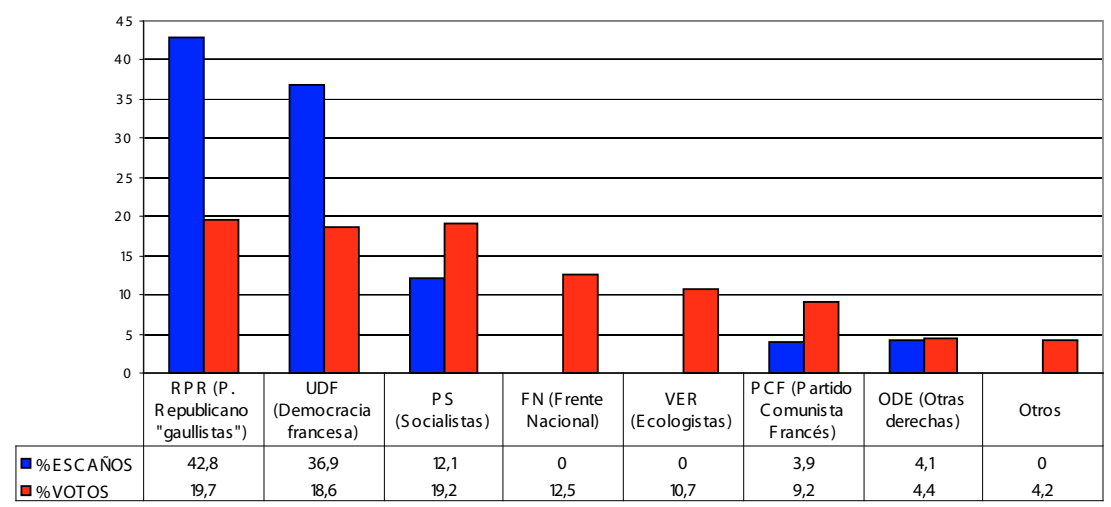

Fuente: Elaboración propia.

\section{I.3. Deformación de hecho en los sistemas mayoritarios}

Los sistemas mayoritarios son propensos a la ingeniería electoral en sus circunscripciones. Puede haber circunscripciones que tengan desigualdad de hecho, por ejemplo: una que tenga 50,000 electores y otra 100,000, pero eligiendo cada circunscripción un representante por igual. Éstos, de una y otra circunscripción, poseen un voto en la Asamblea. Pero la teoría democrática, en vigor, exigiría que el representante de la segunda circunscripción tuviese dos votos frente al de la primera, que sólo debería tener uno.

Estas circunscripciones -suelen ser rurales- facilitan la cooptación por parte de las élites partitocráticas y la generación de feudos electorales.

Otra manipulación se da con el denominado Gerrymander. Se trata de un procedimiento más sutil. Así por ejemplo, en una circunscripción 50,000 electores votan A y 15,000 B; en otra limítrofe, 25,000 electores votan A y 30,000 B. Modificando la frontera de las circunscripciones, se puede hacer pasar 10,000 electores A de la primera a la segunda circunscripción, sin que pasen muchos de B, haciendo así cambiar la mayoría en la segunda circunscripción. Dicho instrumento, como es lógico, puede realizarse donde hay una acentuada distribución del voto por áreas urbanas, por ejemplo en circunscripciones urbanas de los cinturones industriales limítrofes con zonas urbanas de servicios y clase media-alta. 


\section{Sistema PROPORCIONAL}

En nuestra introducción a los sistemas electorales decíamos que los sistemas proporcionales, aunque su nombre indique lo contrario, no guardan una relación voto-escrutinio rigurosa respecto a la voluntad real de los electores. Ésta sólo se daría en el denominado "sistema proporcional integral".

Los sistemas electorales proporcionales tienen una amplia variedad. Pese a esta variedad, podemos decir que la causa por la cual estos sistemas no alcanzan el óptimo democrático del "sistema proporcional integral" es la incorporación de elementos propios de los sistemas mayoritarios. Principalmente se recurre a dos elementos para, por un lado, la atribución local de restos; por otro, el establecimiento de una barrera mínima de votos obligatorios para poder participar en el reparto de escaños.

\section{II.1. Reparto de votos}

El primer paso de los sistemas proporcionales a la hora del recuento es atribuir a cada lista política-electoral los escaños de base que se obtienen según los sufragios que ha reunido, sin tener en cuenta los "restos", es decir, los votos que no pueden repartirse en esta primera fase por los procedimientos matemáticos que a continuación estudiaremos.

Este proceso se puede realizar de tres formas:

a) Con el sistema del "cociente electoral". Éste se obtiene dividiendo en cada circunscripción el número de sufragios expresados entre el número de escaños a otorgar. Obtenido el cociente, una lista tendrá tantos candidatos elegidos cuantas veces el cociente se halle comprendido en la cifra de sufragios obtenidos por las listas.

b) Con el sistema del "número uniforme". Es la ley la que determina el cociente o número de votos que la lista tiene que obtener para conseguir cada representante. Una vez obtenido, una lista tendrá tantos candidatos elegidos cuantas veces el cociente se halle comprendido en la cifra de sufragios obtenidos por las listas; en consecuencia el número de diputados de cada circunscripción no está fijado previamente.

c) Con el sistema del "cociente nacional". Es un sistema intermedio entre los anteriores. El cociente se obtiene dividiendo los sufragios expresados a nivel nacional entre los escaños a cubrir, y una vez determinado el cociente de escaños, éstos se reparten como en el sistema del "número uniforme". 
De estos tres sistemas, el único que se utiliza en la actualidad es el sistema de "cociente electoral", que es sin duda el menos democrático, porque sólo considera los sufragios expresados y no tiene en cuenta ni los votos en blanco ni las abstenciones. Quizá nuestro razonamiento sea tachado de absurdo por muchos politólogos teóricamente demócratas, pero más absurdo resulta que en un Parlamento en el cual debería reflejarse la "voluntad exacta" de los ciudadanos con derecho a voto, no se considere la voluntad de los ciudadanos que no participan del sistema (abstención) o de los que, participando, no aceptan el monopolio partitocrático (voto en blanco). Quizá no sea viable dejar los escaños vacíos, pero sí podría supeditarse el número de escaños a los participantes en las elecciones, ya que ellos y no otros deciden; para eso debería utilizarse un sistema de "número uniforme", como ocurrió en la República de Weimar.

Pero la partitocracia no puede admitir la deslegitimación social patente en las abstenciones y votos en blanco, que llegan en determinadas elecciones a superar el 50\%. De ahí que se elija el sistema del "cociente electoral", ya que éste determina el número de escaños a cubrir siéndole indiferente en número de sufragios expresados por circunscripción.

Tampoco puede ocultarse que este sistema se selecciona porque, frente al del "cociente nacional", aquí el cociente se obtiene para cada circunscripción. Esto permite la existencia de una desigualdad de hecho en el voto individual: el voto de un individuo de una circunscripción poco poblada vale más que el de un individuo de una circunscripción muy poblada, dado que el "coeficiente de reparto" es menor en la primera que en la segunda. Esto ocurre porque los creadores de las leyes electorales no equiparan el número de escaños correspondientes a una circunscripción con la población que tiene esa circunscripción. El motivo es obvio: es más fácil para los partidos con más recursos cooptar votos y establecer feudos electorales donde el coeficiente electoral sea menor. Por ejemplo, en España, un diputado se consigue en Soria con poco más de 30,000 votos, mientras que en Madrid suelen ser necesarios más de 200,000 votos. Estas cifras varían dependiendo de la abstención, votos en blanco y nulos (por defecto de forma o falta de computación).

\section{II.2. Reparto de restos}

Tras el reparto inicial de escaños, entramos en la segunda fase: el reparto del resto, es decir, el reparto de los votos que no fueron (en la primera fase) 
lo cuantitativamente numerosos para alcanzar la cifra del cociente electoral. Si una lista obtuvo 86,000 votos y el "cociente electoral" fue 35,000, la lista electoral se habría atribuido (en la primera fase) dos escaños; se trata ahora de ver cómo se reparten los 16,000 votos restantes (restos).

Para realizar esta operación hay fundamentalmente tres procesos matemáticos:

a) Atribución de restos al "resto más amplio". Según este sistema, los escaños no provistos se atribuyen al resto más grande, teniendo en cuenta los restos tal y como quedan después de la primera fase de reparto (la del cociente electoral). Este método equilibra el reparto, ya que los partidos minoritarios, al haber quedado por debajo del coeficiente electoral encuentran convertidos todos sus sufragios en restos y en consecuencia, tienen medias elevadas.

b) Atribución de restos a la "media más elevada". Esta media se obtiene de una operación donde el dividendo es la cifra de votos total. Con ello se prima a los partidos mayoritarios frente a los minoritarios, ya que su media será más elevada. Este beneficio que se otorga a los grandes partidos se produce con idénticos resultados en el método d'Hont, pero el procedimiento a seguir es diferente. Este método tiene la peculiaridad de que la natural sobrerrepresentación del partido grande se acentúa cuando hay más partidos (para repartir) y menos sufragios emitidos (votos a repartir). Este sistema se aplica en España, siendo sus efectos más exagerados al aplicarse a la totalidad de los votos (en cada circunscripción). Así, los procesos del reparto de votos y restos son sustituidos por el sistema d'Hont (vid. gráfico 3). 
Gráfico 3: Sistema electoral proporcional. España: elecciones generales del 6 de junio de 1994

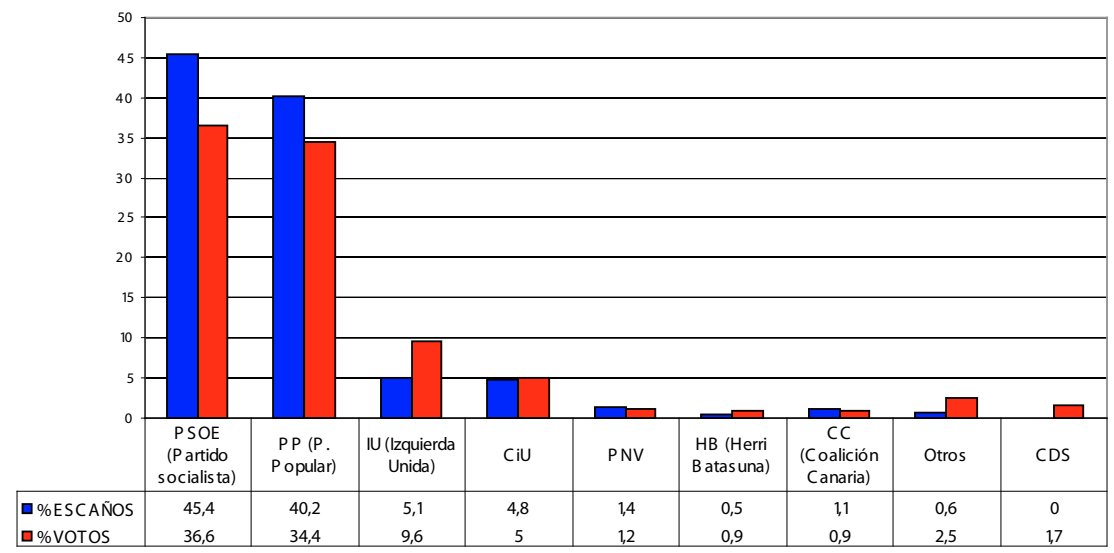

Fuente: Elaboración propia.

Gráfico 3-bis: Sistema electoral proporcional. España: elecciones generales del 14 de marzo de 2004

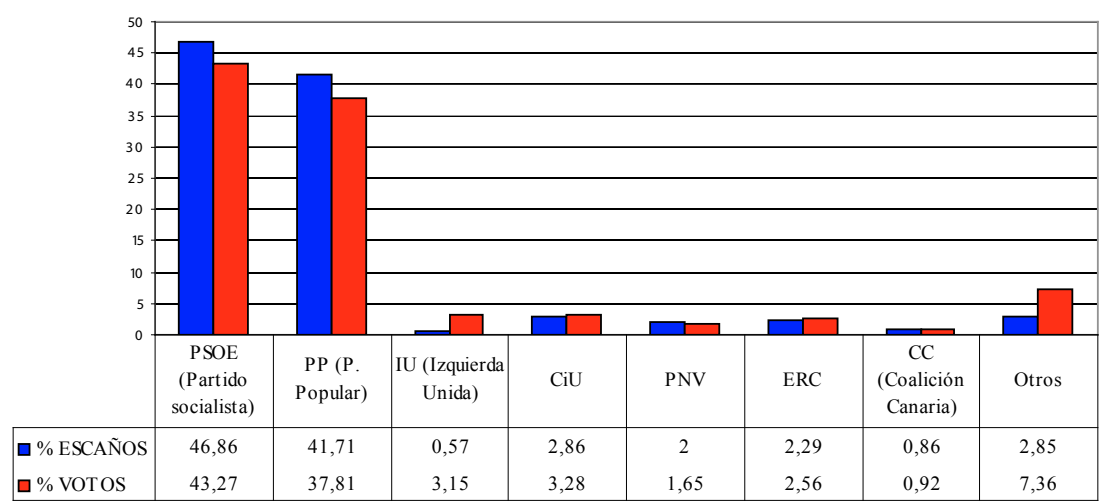

Fuente: Elaboración propia.

c) Atribución de restos por medio del "coeficiente rectificado", también llamado "sistema Hagenbach-Bischof". Este sistema disminuye el cociente electoral (para el reparto de escaños en la primera fase) a la hora de repartir los restos. Esta disminución se produce añadiendo ficticiamente un escaño al dividendo que se aplica. Así se atribuirá un escaño. El proceso se repite hasta que se reparten todos los escaños. En teoría, este sistema suaviza las repercusiones del método d'Hont porque, al reducir el coeficiente electoral, 
facilita el acceso real de los partidos minoritarios. Este sistema era, con algunos matices, el vigente en Italia antes de la última reforma electoral general (vid. gráfico 4). En concreto, había dos características que lo hacían más proporcional: en primer lugar, el coeficiente rectificado se aumentaba en dos escaños ficticios y no en uno; además había un número importante de restos que se repartían a nivel nacional y no en la circunscripción.

Gráfico 4: Sistema electoral proporcional. Italia: elecciones generales de 6 de abril de 1992

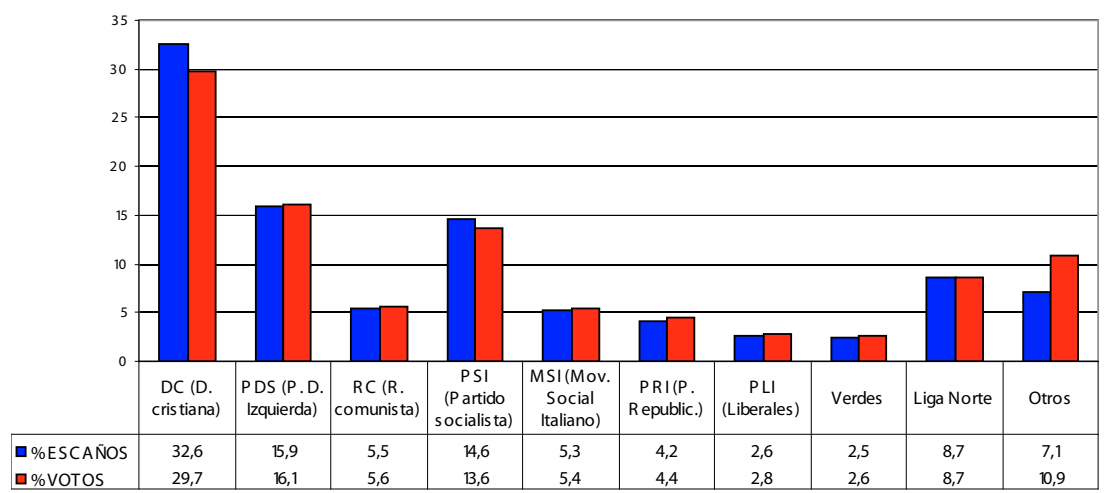

Fuente: Elaboración propia.

\section{II.3. Critica cualitativa al sistema proporcional}

Visto el funcionamiento genérico de los sistemas proporcionales, es teóricamente lógico que, desde un punto de vista democrático, se le defienda como sistema idóneo. Si insistimos en las críticas cuantitativas anteriores, referidas al rigor de reparto, es necesario decir que los sistemas proporcionales aumentan la discrecionalidad de las élites de los partidos mayoritarios. En los sistemas proporcionales, las listas de los partidos son elaboradas por la élite, que sitúa en ellas a quien desea, para articular sus relaciones de poder dentro del partido y para garantizar su propio estatus. ${ }^{2}$ $\mathrm{Al}$ ser listas cerradas y bloqueadas, salvo raras excepciones, el ciudadano no puede no elegir a quien no quiera como representante, pudiendo las élites ir camufladas en las listas y forzando así su elección. En el caso de que hubieran listas abiertas, como en Italia, se hacían sólo en las circuns-

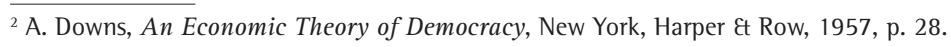


cripciones, dejando la lista nacional cerrada. Y en aquellos países que admiten listas abiertas (como en Suiza), prácticamente no son utilizadas por la falta de información y conocimiento. Pero esto nos parece de importancia menor. Como mostramos, la élite partitocrática no necesita estar en el Parlamento para determinar lo que allí se hace.

\section{Sistemas mixtos}

Como su propio nombre indica, estos sistemas surgen de la interrelación de los sistemas mayoritario y proporcional. El atractivo que se encuentra en él, es el de permitir un paso intermedio que supera los problemas de fragmentación parlamentaria tal y como se produce con un sistema proporcional. Es una manera eficaz de eliminar a las minorías del juego parlamentario sin que resulte tan flagrante como en los sistemas mayoritarios.

Prototípico ejemplo es el caso alemán. Los electores votan con dos papeletas: una sirve para elegir a la mitad de los diputados por el escrutinio uninominal a una sola vuelta (se rellena la papeleta con el nombre del candidato individual); la otra se rellena con el nombre del partido. Estas segundas papeletas sirven para calcular proporcionalmente (sistema Nimeyer) el número total de escaños que cada partido habría obtenido de la representación proporcional. De este número se deduce el de diputados ya obtenidos por el partido a través de los votos individuales. Si estos últimos le han concedido más escaños que los que debería haber tenido por la representación proporcional, los conserva. Esto es lo que introduce la prima a la mayoría.

Esa prima no es abultada (vid. gráfico 5). Pero es necesario apreciar que el sistema alemán introduce una "barrera mínima" necesaria del 5\% de votos a nivel nacional para entrar en el reparto de escaños. Esta barrera provoca que el número de votos que entran como computables en el reparto proporcional sea mucho menor de lo que debería ser teóricamente. Si bien los votos que desecha este 5\% son menores que los que desecha el sistema mayoritario, lo cierto es que tal práctica hace que la diferencia entre ambos sistemas sea menor. Con ello se tiende a reducir las posibilidades de fragmentación del Parlamento pero a costa de dificultar el surgimiento y mantenimiento de partidos minoritarios o alternativos.

Tras la unificación alemana, un partido con más de tres millones de votos puede ser extraparlamentario. Así, los Grünen (Partido Verde) pasaron de tener 42 escaños en 1987 a no tener ninguno tras la unificación. La 
barrera hizo que el Tribunal Constitucional alemán declarase que no podía aplicarse la norma a los estados federales del Este en la primera elección tras la unificación.

\section{Gráfico 5: Sistema electoral mixto. Alemania: elecciones generales del 2 de} diciembre de 1990

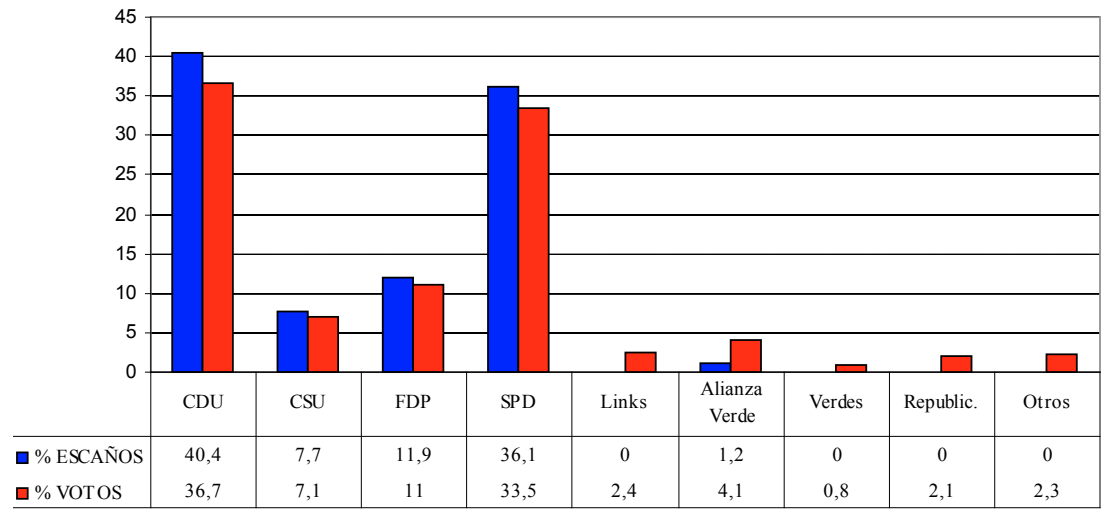

Fuente: Elaboración propia.

Gráfico 5-bis: Sistema electoral mixto. Alemania: elecciones generales del 18 de septiembre de 2005

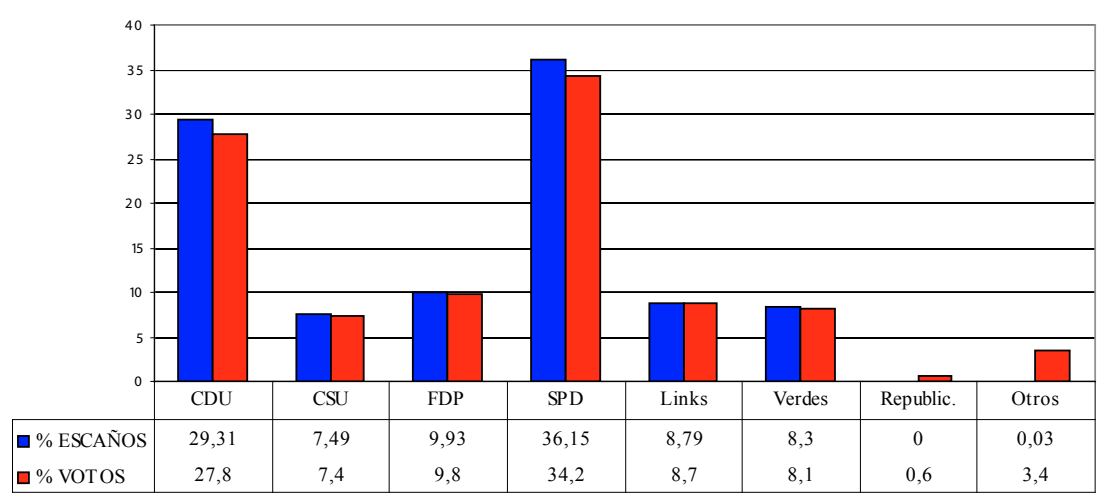

Fuente: Elaboración propia. 


\section{LAS FUtURAS REFORMAS ELECTORALES}

Los sistemas electorales rara vez son neutrales, suelen tender a favorecer a sus promotores que son juez y parte, los grandes partidos, sin reflejar la realidad de la sociología electoral. Ninguno es estrictamente proporcional, traicionando así el ideal democrático. Cuanto más democrático es un sistema electoral, es decir, cuanto más se aproximen las preferencias de los electores a la composición del Parlamento, más se fragmenta este último, dándose consecuentemente con mayor facilidad escenarios de ingobernabilidad del Parlamento por parte de los ejecutivos a través del sistema de partidos; si se prefiere, en sentido contrario, dándose escenarios de mayor autodeterminación parlamentaria.

Frente a esta realidad, los partidos mayoritarios buscan, ingeniería electoral mediante, introducir características mayoritarias en sistemas proporcionales, alejándolos más del referente del sistema proporcional integral. La finalidad última sería sustituir el sistema proporcional por el mayoritario, por ser éste más acorde con los intereses de los denominados partidos de gobierno. No podemos dudar de la capacidad de la "electometría partitocrática" para generar mayorías parlamentarias que alteren la realidad sociopolítica. Las últimas reformas electorales adoptadas en Europa apuntan en ese sentido, mostrándonos vías para realizar este proceso.

Tanto el sistema electoral ruso como el actual sistema italiano pueden calificarse técnicamente de mixtos, pero de facto su realidad viene mucho más marcada por la lógica mayoritaria que, por ejemplo, el sistema alemán considerado paradigma de sistema electoral mixto. La predicha realidad no parece encontrarse en el óptimo democrático, sobre todo si tenemos en cuenta que las primeras elecciones en las que se aplicaron intentaron marcar una nueva era política cuya suerte, sin embargo, estuvo viciada de principio.

\section{IV.1. El sistema ruso}

El actual sistema electoral ruso ${ }^{3}$ establece un sistema mixto de elección de los representantes en la Duma estatal. Según este sistema, el 50\% de los representantes son elegidos por los distritos electorales federales, bajo

${ }^{3}$ Datos y referencias normativas en Keesing's Record World Evens, Volumen 39, número 12, año 1993. 
la lógica proporcional; la otra mitad, por sistema mayoritario uninominal a través de 225 distritos electorales formados por provincias, territorios, repúblicas, unidades nacionales y ciudades de significación federal.

El sistema proporcional tiene un sesgo mayoritario al no incorporar en el recuento a las listas que no superen el 5\%, como en el caso alemán. Así, el 13.3\% de votos que suman dichas listas son despreciados, lo cual provoca un aumento en el porcentaje de escaños respecto al de votos de las listas que superan el umbral. Pero es la mitad mayoritaria del sistema la que introduce el mayor desequilibrio, de suerte que el partido más votado, PLDR, con más del 7\% sobre Cambio de Rusia, sucumbe en la suma total en casi el 6\% (vid. gráfico 6). El sistema mayoritario prima a los partidos de concentración de voto -Cambio de Rusia y PCR- en detrimento de los que, teniendo un mayor apoyo total, lo distribuyen sin embargo por toda la geografía, como es el caso del PLDR y Mujeres de Rusia, fundamentalmente.

Esta potencialidad genérica, se ve ampliada por la desproporción poblacional de las circunscripciones, dada la diversidad de entes territoriales que forman circunscripciones. La ley permite una desproporción del 15\%, con lo que ello supone en un país con 137,552,000 habitantes. Con dicha población y una extensión geográfica de 17,075,400 kilómetros cuadrados, divididos solamente en 225 circunscripciones mayoritarias, podemos afirmar que las desproporciones son asimilables a las de un sistema mayoritario.

Gráfico 6: Sistema electoral mixto. Rusia: elecciones generales del 12 de diciembre de 1993

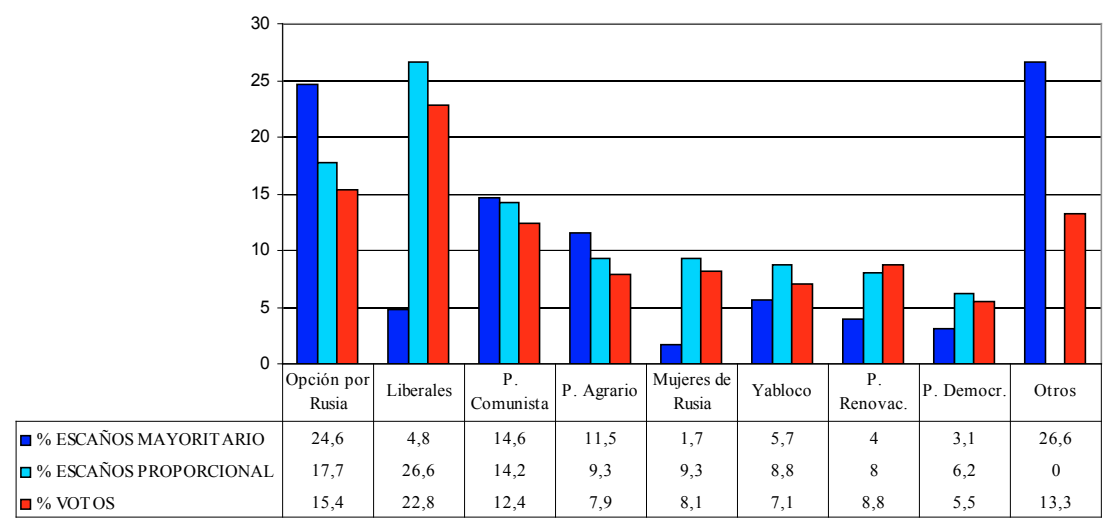

Fuente: Elaboración propia. 


\section{IV.2. El sistema italiano}

El actual sistema electoral italiano ${ }^{4}$ establece un sistema mixto de elección: el 75\% de la Cámara de Diputados, 425 escaños, se elige en otras tantas circunscripciones con el sistema mayoritario uninominal; el 25\%, 155 de los 630 escaños, por el proporcional con base regional.

El proporcional margina a las listas regionales que no superan el 4\%. Aunque este mecanismo proporcional tenía por objeto ser un respiro para las listas desprotegidas en el sistema mayoritario (pequeños partidos), de hecho, es poco más que un refugio para los líderes fracasados en su enfrentamiento uninominal, ya que cuentan con la protección de las listas proporcionales. La lógica mayoritaria reparte 159 escaños más de los necesarios para alcanzar la mayoría absoluta, lo cual juega desequilibrando la balanza del lado mayoritario.

Tras el diseño del sistema, no pocos afirmaron que la fragmentación en Italia es tan intrínseca que resulta inevitable, por muy mayoritario que sea el sistema electoral. Pero no debemos engañarnos: los sistemas electorales no entienden de coaliciones preelectorales. Hoy ya podemos hablar de la incidencia real de este sistema electoral en el sistema de partidos hasta el punto de que dichos partidos nunca se llegaron a enfrentar de forma individual, fuera de coaliciones electorales. Los partidos, por las inseguridades propias y por las ansias de poder, entraron en una maraña de coaliciones que ha consolidado dos grandes bloques difuminadores de buena parte de los perfiles ideológicos de los partidos, véase si no la evolución de AN y el PCI-PDI hasta nuestros días. Dicho proceso no sólo nos impide apreciar el apoyo real de los partidos, al no dejar al electorado decidir por un partido (salvedad hecha de las candidaturas independientes), ${ }^{5}$ sino que demuestra la consolidación de la difuminación de las fracturas ideológico-electorales.

Independientemente de la incidencia clara en el actuar de los partidos del sistema electoral, las "virtudes" del sistema quedaron demostradas desde las primeras elecciones donde los dos bloques principales consiguieron

${ }^{4}$ Referencias normativas: Supplemento ordinario alla Gazzetta Ufficiale, 6, 10 de junio de 1994, Serie Generale y Guida alle Eleziolli, publicado por I1 Sole los días 11, 20, 21, 27 y 28 de febrero de 1994. Datos en Corriere della Sera y La Repubblica, 20 y 30 de marzo, 1994.

${ }^{5}$ Esto se puso de manifiesto desde las primeras elecciones, donde por ejemplo se enfrentaron líderes de partidos que concurrieron coaligados en diferentes circunscripciones: en el colegio 1 de Milán, Bossi, apoyado por la Liga y Forza Italia, se enfrentó a La Russa, de Alianza Nacional; igual ocurrió en el colegio 4 de Turín. 
acaparar el 91.8\% de los escaños con solamente el 72.3\% de los votos, siendo el Polo de la Libertad (la fuerza) el más beneficiado con 12 puntos, seguido del progresista ( $2^{\text {a fuerza) }}$ con más de 7 y penalizando con 8 puntos a la coalición de centro ( $3^{\text {a }}$ fuerza) (vid. gráfico 7). La evolución -pese al matiz de ese $25 \%$ proporcional- es paralela al sistema inglés, lo que nos autoriza a afirmar que el sistema italiano incorpora las desigualdades de hecho de los sistemas mayoritarios.

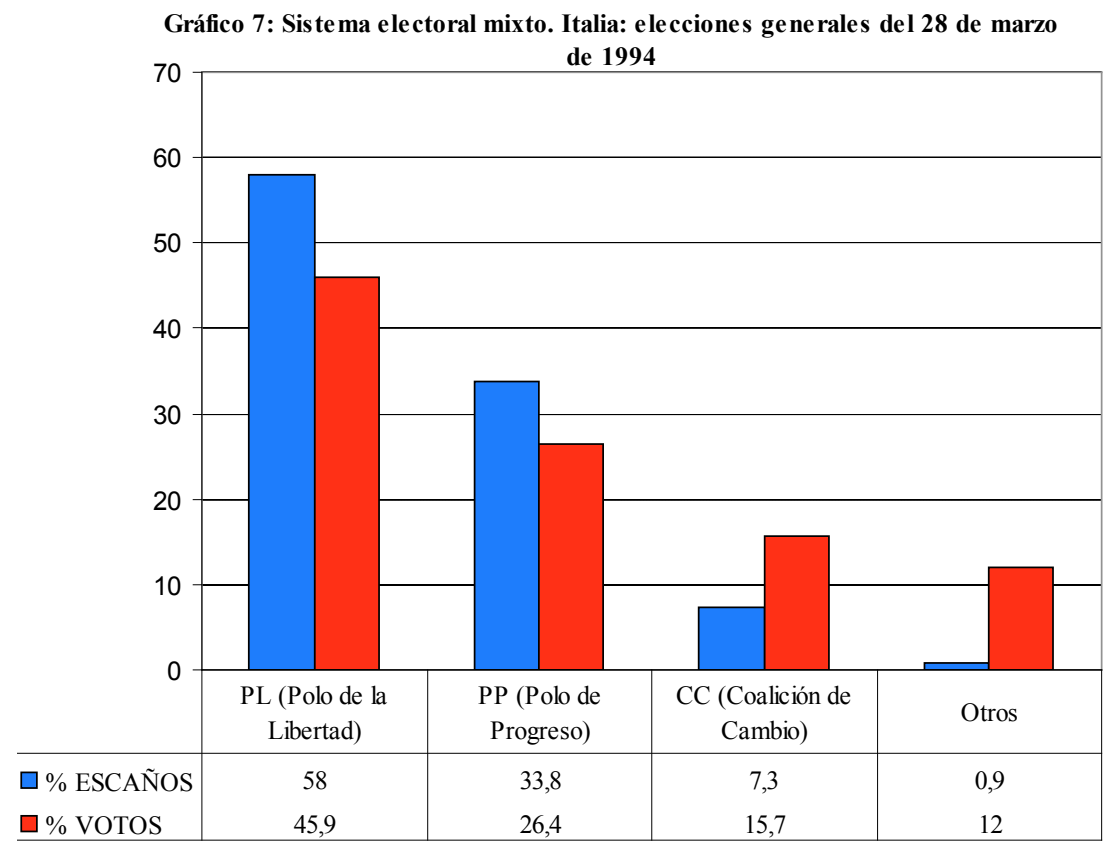

Fuente: Elaboración propia. 


\section{Referencias}

Abrams, B.A., "Political Power and the Market for Governors", Public Choice, 37, 1981, 521-529.

y Settle, R. F., "Campaign-Finance Reform: A Public Choice Perspective”, Public Choice, 120, 2004, 379-400.

Ansolabehere, S., De Figueiredo, J. y Snyder, J.M., "Why Is There So Little Money in U.S. Politics?”, Journal of Economic Perspectives, 17, 2003, 105-130.

Bronaronnars, S. y Lott J. Jr., "Do Campaign Contributions Alter How Politicians Votes? Or Do Donors Support Candidates Who Value the Same Things That they Do?", Journal of Law and Economics, 40, 1997, 317-350.

Cotteret, J.M. y Emeri C., Los sistemas electorales, Oikos-Tau, Barcelona, 1973.

Crain, W.M., Deaton, T. y Tollison, D., "Legislators as Taxicabs: On the Value of Seats in the U.S. House of Representatives”, Economic Inquiry, april, 1977, 298-302.

, Tollison, D., "Attenuated Property Rights and the Market for Governors", Journal of Law and Economics, april, 1977, 205-211.

Douglas W.R., Leyes electorales y sistemas de partidos, Editorial Citep, 1977.

Downs, A., An Economic Theory of Democracy, New York, Harper \& Row, 1957.

Duverger, M., Instituciones politicas y derecho constitucional, Ariel, Barcelona, 1988.

Finer, S.E., Politica de adversarios y reforma electoral, Fondo de Cultura Económica, México, 1980.

Gifford, A. y Santoni, G.J., "Politicians and Property Rights: An Analysis of the Relationship Between Campaign Expenditures and Wage and Price Controls", Public Choice, 33, 1978, 71-74.

Lott, J. Jr., “A Simple Explanation Why Campaign Expenditures Are Increasing: The Government is Getting Bigger", Journal of Law and Economics, 43, 2000, 359-393.

Nohlen, D., Los sistemas electorales del mundo, Centro de Estudios Constitucionales, Madrid, 1981.

Palda, F., "The Determinants of Campaign Spending: The Role of the Government Jackpot”, Economic Inquiry, 30, 1992, 627-638.

Stratmann, T., "What Do Campaign Contributions Buy?: Deciphering Causal Effects of Money and Votes”, Southern Economic Journal, 57, 1991, 606-620.

Volver al Índice>> 